\title{
The Serum Creatinine Level Might Be Associated with the Onset of Impaired Fasting Glucose: A Community-based Longitudinal Cohort Health Checkup Study
}

\author{
Naohiko Yoshida ${ }^{1}$, Teruki Miyake ${ }^{2}$, Shin Yamamoto ${ }^{2}$, Shinya Furukawa ${ }^{3,4}$, Hidenori Senba ${ }^{2}$, \\ Sayaka Kanzaki ${ }^{2}$, Mitsuhito Koizumi ${ }^{2,5}$, Toru Ishihara ${ }^{2,5}$, Osamu Yoshida ${ }^{2}$, Masashi Hirooka ${ }^{2}$, \\ Teru Kumagi ${ }^{6}$, Masanori $\mathrm{Abe}^{2}$, Kohichiro Kitai ${ }^{5}$, Bunzo Matsuura ${ }^{2}$ and Yoichi Hiasa ${ }^{2}$
}

\begin{abstract}
:
Objective Skeletal muscle is the main target organ for glycemic control, and the serum creatinine level is a convenient indicator of the skeletal muscle mass. This study aimed to assess the potential relationship between the serum creatinine level and the onset of impaired fasting glucose (IFG).

Methods In this large, community-based, retrospective longitudinal cohort study, we examined the records of 7,905 Japanese participants (3,863 men, 4,042 women) of 18-80 years of age who underwent annual health checkups at a single center between April 2003 and August 2013. After applying the exclusion criteria, 6,490 participants were reviewed to identify those with the onset of IFG, defined as a fasting plasma glucose $\geq 6.11 \mathrm{mM}$. Among the participants, 278 met the criterion for the onset of IFG during the observation period. Results Creatinine levels were higher in male subjects who exercised periodically and were exercise conscious in comparison to those who did not exercise, and were higher in female subjects who exercised periodically in comparison to female subjects who did not exercise and who were not exercise conscious. Additionally, the serum creatinine level was negatively associated with the onset of IFG in both men [adjusted hazard ratio, 0.98; 95\% confidence interval (CI), 0.96-0.99; $\mathrm{p}=0.008$ ] and women (adjusted hazard ratio, 0.94; $95 \% \mathrm{CI}, 0.91-0.97 ; \mathrm{p}<0.001$ ) after adjustment for variables previously reported to be risk factors for the onset of glucose intolerance and factors associated with chronic kidney disease.

Conclusion A low creatinine level might be associated with the onset of IFG. Moreover, the fact that serum creatinine levels increase with exercise might demonstrate the importance of exercise therapy.
\end{abstract}

Key words: serum creatinine, impaired fasting glucose, community-based longitudinal cohort study

(Intern Med 58: 505-510, 2019)

(DOI: 10.2169/internalmedicine.0760-18)

\section{Introduction}

Type 2 diabetes is a public health concern worldwide that can lead to macro- and microvascular complications as well as nonalcoholic steatohepatitis and bone metabolism defects (1-3). Although effective early glycemic control is nec- essary to prevent the progression of complications, the majority of type 2 diabetes patients have no symptoms and are sometimes only diagnosed after the development of serious complications. Thus, various predictive markers for abnormal glucose tolerance are needed to detect the onset of diabetes earlier.

Skeletal muscle, which accounts for $80 \%$ of the glucose

\footnotetext{
${ }^{1}$ Department of Internal Medicine, Ozora Hospital, Japan, ${ }^{2}$ Department of Gastroenterology and Metabology, Ehime University Graduate School of Medicine, Japan, ${ }^{3}$ Department of Epidemiology and Preventive Medicine, Ehime University Graduate School of Medicine, Japan, ${ }^{4}$ Epidemiology and Medical Statistics Unit, Translational Research Center, Ehime University Hospital, Japan, ${ }^{5}$ Ehime General Health Care Association, Japan and ${ }^{6}$ Department of Community Medicine, Ehime University Graduate School of Medicine, Japan Received: January 2, 2018; Accepted: July 30, 2018; Advance Publication by J-STAGE: October 17, 2018 Correspondence to Dr. Teruki Miyake, teruki-ygc@umin.ac.jp
} 


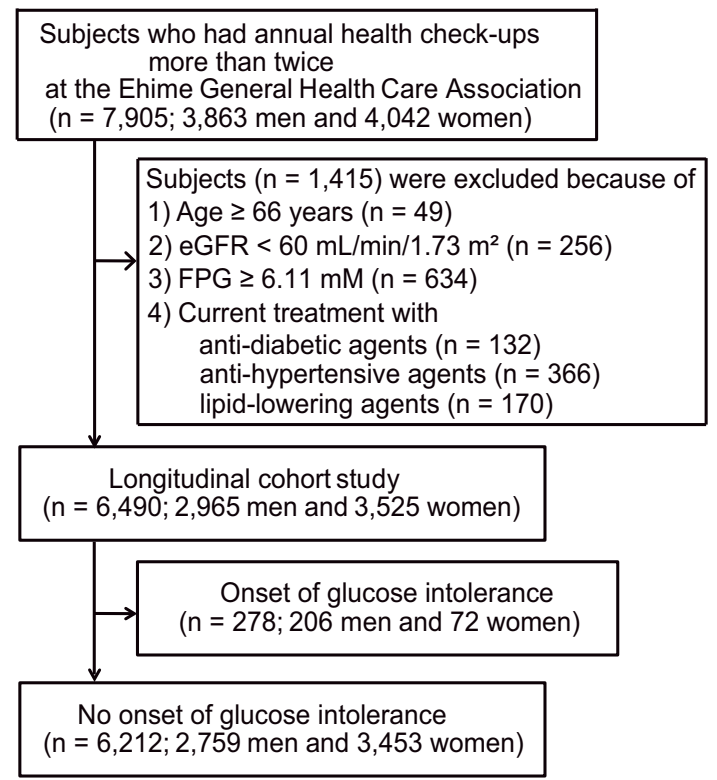

Figure 1. A flowchart of participant recruitment. eGFR: estimated glomerular filtration rate

uptake under euglycemic and hyperinsulinemic conditions, takes up glucose via GLUT4 in response to insulin stimulation (4). Skeletal muscle, therefore, comprises the largest insulin-sensitive organ in the human body, and as such, is the main target organ for glycemic control $(5,6)$. The association between the skeletal muscle mass and glucose intolerance has been investigated in several population-based studies $(4,7,8)$, and in a few cross-sectional studies, patients with diabetes were found to have a greater muscle mass than those without diabetes after accounting for body size $(7,8)$. However, Kim et al. reported that the skeletal muscle mass (adjusted for height or weight) was significantly lower in patients with diabetes than in those without diabetes in the Korean Sarcopenic Obesity Study (KSOS), which included 414 patients with diabetes and 396 controls (9). Furthermore, insulin resistance in skeletal muscle precedes $\beta$-cell failure and hyperglycemia and plays a key role in the development of type 2 diabetes (4). Thus, the assessment of the skeletal muscle mass might help predict the onset of type 2 diabetes.

Creatinine, which is a metabolite of creatine and creatine phosphate, is produced in the skeletal muscle at a relatively constant rate (10). Creatinine level reflects the absolute amount of skeletal muscle and is therefore a useful indicator of the skeletal muscle mass (11). Thus, in this large, community-based, retrospective longitudinal cohort study, we aimed to examine the association between serum creatinine levels and the onset of impaired fasting glucose (IFG). The results of this study will assist in identifying patients who are at high risk of developing type 2 diabetes and providing interventions to mitigate the onset of type 2 diabetes.

\section{Materials and Methods}

In the present study, data were extracted from the medical records of 7,905 Japanese subjects (3,863 men, 4,042 women; age range, 18-80 years) who had undergone more than two annual health checkups between April 2003 and August 2013 at the Ehime General Health Care Association. Details of these data have been described in our previous report (12). Briefly, the data contained findings from physical examinations, anthropometric measurements and other routine biochemical variables, personal medical histories and second-degree family histories of diabetes, prescribed medications, exercise habits (no exercise, exercise consciousness, or periodic exercise), as well as the quantity and frequency of alcohol intake (alcohol consumption: alcohol intake $\geq 30$ $\mathrm{g} /$ day for men and $\geq 20 \mathrm{~g} /$ day for women) (13) and cigarette smoking. Fasting blood samples were used to measure the levels of the following parameters: creatinine; fasting plasma glucose; lipid profile; uric acid; and alanine aminotransferase. The onset of IFG was diagnosed based on a fasting plasma glucose level of $\geq 6.11 \mathrm{mM}$ in any of the annual health checkups $(14,15)$. The equation of the Japanese Society of Nephrology was used to calculate the estimated glomerular filtration rate (16).

This study protocol was approved by the Committee for Medical Ethics of Ehime University Hospital (Approval ID No. 110405, University Hospital Medical Information Network ID No. UMIN000011953) and was conducted in accordance with the tenets of the Declaration of Helsinki, 1975 and its later amendment in 1983.

As shown in Fig. 1, out of the 7,905 reviewed participants, 1,415 were excluded based on the following exclusion criteria: age $\geq 66$ years $(n=49)$; estimated glomerular filtration rate $<60 \mathrm{~mL} / \mathrm{min} / 1.73 \mathrm{~m}^{2}(\mathrm{n}=256)$; fasting plasma glucose $\geq 6.11 \mathrm{mM}(\mathrm{n}=634)$; and/or current treatment with antidiabetic $(n=132)$, antihypertensive $(n=366)$, or lipidlowering agents $(n=170)$. After applying the exclusion criteria, the final study group consisted of 6,490 reviewed participants (2,965 men, 3,525 women). The mean observation period was $4.38 \pm 2.52$ years (median, 4.05 years; range, 0.44-10.13 years; men, $4.28 \pm 2.52$ years, women, $4.45 \pm 2.53$ years). The median interval between visits was 1.04 years (range, 0.44-9.05 years); this value was $<6$ months and $>2$ years for $0.34 \%$ and $18.2 \%$ of the participants, respectively.

To maintain anonymity, a numerical code was assigned to each participant and all data were maintained in a secure database. The requirement to obtain informed consent from the participants was waived because of the retrospective nature of the study. Statistical analyses were performed using the JMP (version 12; SAS Institute Japan, Tokyo, Japan) and XLSTAT (Addinsoft, Paris, France) software programs. The Mann-Whitney U test was used to compare differences between men and women in baseline characteristics, and the chi-squared test was used to compare associations between health-related behavior and a family history of diabetes. The 
Table 1. Characteristics at Baseline and Impaired Fasting Glucose Onset.

\begin{tabular}{llccc}
\hline & & $\begin{array}{c}\text { Men } \\
(\mathrm{n}=2,965)\end{array}$ & $\begin{array}{c}\text { Women } \\
(\mathrm{n}=3,525)\end{array}$ & $\mathrm{p}$ value \\
\hline Age & $($ years $)$ & $41.9 \pm 8.6$ & $40.4 \pm 8.5$ & $<0.001$ \\
Body mass index & $\left(\mathrm{kg} / \mathrm{m}^{2}\right)$ & $23.5 \pm 3.1$ & $21.3 \pm 3.1$ & $<0.001$ \\
Systolic blood pressure & $(\mathrm{mmHg})$ & $115 \pm 14$ & $107 \pm 15$ & $<0.001$ \\
Alanine aminotransferase & $(\mathrm{U} / \mathrm{L})$ & $27.8 \pm 20.4$ & $16.0 \pm 9.7$ & $<0.001$ \\
Serum creatinine & $(\mu \mathrm{M})$ & $76.1 \pm 9.0$ & $55.5 \pm 7.4$ & $<0.001$ \\
Uric acid & $(\mu \mathrm{M})$ & $367 \pm 71$ & $252 \pm 52$ & $<0.001$ \\
Fasting plasma glucose & $(\mathrm{mM})$ & $5.22 \pm 0.37$ & $4.91 \pm 0.39$ & $<0.001$ \\
Total cholesterol & $(\mathrm{mM})$ & $5.24 \pm 0.87$ & $5.21 \pm 0.88$ & 0.055 \\
Triacylglycerol & $(\mathrm{mM})$ & $1.49 \pm 1.16$ & $0.86 \pm 0.52$ & $<0.001$ \\
Alcohol drinkers & $(\%)$ & 28.1 & 4.9 & $<0.001$ \\
Current smokers & $(\%)$ & 42.1 & 5.65 & $<0.001$ \\
Exercise habit* & Periodic exercise $(\%)$ & $34.5 \%$ & $25.3 \%$ & $<0.001$ \\
Family history of diabetes & $(\%)$ & 16.7 & 22 & $<0.001$ \\
Onset of impaired fasting glucose $* *$ & $(\%)$ & 6.95 & 2.04 & $<0.001$ \\
\hline
\end{tabular}

Values are expressed as means \pm standard deviations or percentages $(\%)$. The p values were determined using the Mann-Whitney $U$ test or chi-squared test, as appropriate.

*Exercise habit: no exercise or consciousness of exercise vs. periodic exercise.

**The onset of impaired fasting glucose was defined as a fasting plasma glucose level $\geq 6.11 \mathrm{mM}$ during the observation period.

Steel-Dwass test was employed to analyze between-group differences in baseline exercise habits, and a univariate Cox proportional hazard regression model with forward likelihood ratio tests was used to assess the importance of each variable related to the incidence of the onset of IFG and chronic kidney disease. A multivariate Cox proportional hazard regression model was used to calculate the adjusted hazard ratios (aHRs) of serum creatinine for the incidence of the onset of IFG, which were adjusted for the following factors known to be related to glucose intolerance and chronic kidney disease $(12,17-20)$ : age, body mass index, systolic blood pressure, uric acid, fasting plasma glucose, exercise habits, and a family history of diabetes (Model 1). Model 2 was adjusted for variables associated with metabolic disease, including body mass index, systolic blood pressure, and the levels of alanine aminotransferase, uric acid, fasting plasma glucose, total cholesterol, and triacylglycerol. Model 3 was adjusted for variables associated with lifestyle, alcohol consumption, current smoking status, and exercise habits. All reported $\mathrm{p}$ values were two-tailed, and $\mathrm{p}$ values of $<0.05$ were considered to indicate statistical significance.

\section{Results}

\section{Baseline characteristics}

All variables could be used in our review with the exception of the BMI of one participant $(0.015 \%)$. In comparison to the women, the men in this study were older and had significantly higher values for metabolic and serum markers other than total cholesterol $(\mathrm{p}<0.001$ for each variable; Table 1). Additionally, the frequency of alcohol consumption, current smoking, and periodic exercise was higher among men, while fewer men had a family history of diabetes $(\mathrm{p}<$ 0.001 for each variable; Table 1). The onset of IFG was more frequent in men than in women $(p<0.001$; Table 1$)$. Moreover, the creatinine levels were higher in male subjects who exercised periodically ( $<<0.001$; Fig. 2) and were exercise conscious ( $p=0.041$; Fig. 2 ) than in those who did not exercise, and were higher in female subjects who exercised periodically in comparison to female subjects who did not exercise $(\mathrm{p}<0.001$; Fig. 2$)$ and who were not exercise conscious ( $<<0.001$; Fig. 2).

\section{Risk factors for the onset of IFG}

The univariate analyses showed significant positive associations of the following variables with the onset of IFG: serum creatinine [men: hazard ratio (HR), 0.975; 95\% confidence interval (CI), 0.96-0.99; $\mathrm{p}=0.001$; women: HR, 0.967; 95\% CI, 0.937-0.999; $\mathrm{p}=0.04$; Table 2], previously reported risk factors for IFG, and metabolic disease-related factors (men: age, BMI, systolic blood pressure, alanine aminotransferase, uric acid, fasting plasma glucose, total cholesterol, triacylglycerol, current smoking status, and family history; women: age, BMI, systolic blood pressure, alanine aminotransferase, uric acid, fasting plasma glucose, total cholesterol, triacylglycerol, and family history; Table 2).

\section{Serum creatinine as a risk factor for the onset of IFG}

The multivariate analysis was adjusted for previously reported risk factors for the onset of glucose intolerance and factors associated with chronic kidney disease. Model 1 suggested significantly positive associations between serum creatinine levels and the onset of IFG in both sexes (men: aHR, 0.98; 95\% CI, 0.96-0.99; $\mathrm{p}=0.008$; women: aHR, 0.94; $95 \%$ 
Serum creatinine

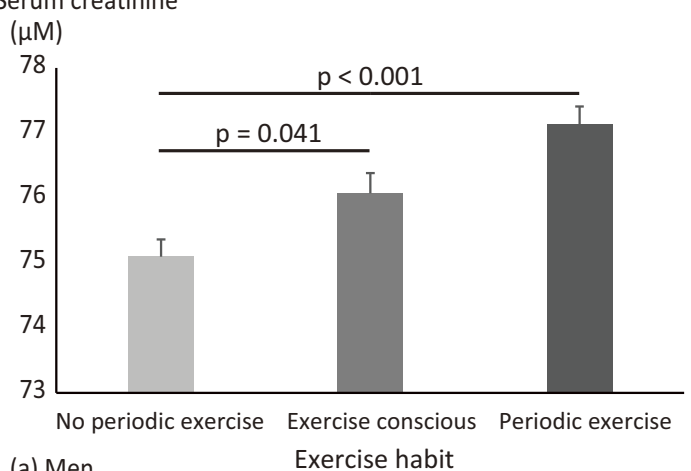

(a) Men

Serum creatinine

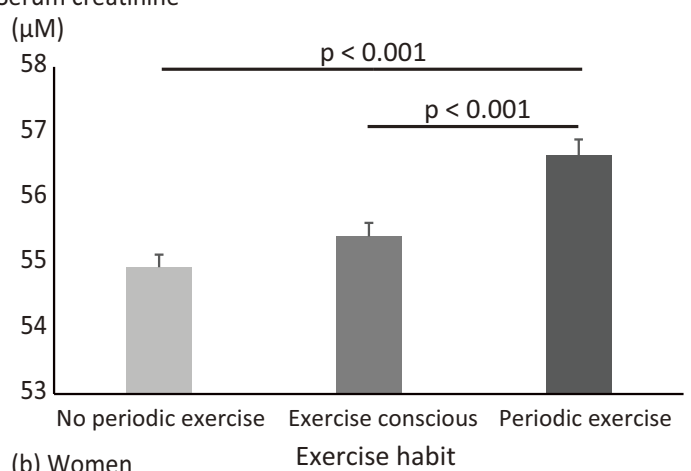

(b) Women

Figure 2. The serum creatinine levels according to exercise habits and sex. Among men, creatinine levels were higher in subjects who exercised periodically $(\mathbf{p}<0.001)$ and were exercise conscious ( $p=0.041$ ) compared to subjects who did not exercise. On the other hand, among women, subjects who exercised periodically had higher creatinine levels in comparison to subjects who did not exercise $(\mathbf{p}<0.001)$ and who were not exercise conscious $(\mathbf{p}<\mathbf{0 . 0 0 1})$. The $p$ values were determined using the Steel-Dwass test.
CI, 0.91-0.97; p<0.001; Table 3). Model 2, which was adjusted for variables associated with metabolic disease and Model 3 which was adjusted for variables associated with lifestyle, also revealed significant associations between serum creatinine levels and the onset of IFG (Model 2: men: aHR, 0.98; 95\% CI, 0.96-0.99; $\mathrm{p}=0.002$; women: aHR, 0.94; 95\% CI, 0.92-0.97; p<0.001) (Model 3: men: aHR, 0.98; 95\% CI, 0.96-0.99; p=0.004; women: aHR, 0.967; 95\% CI, 0.937-0.999; $\mathrm{p}=0.041$ ) (Table 3).

\section{Discussion}

The present community-based study was conducted to examine the association between serum creatinine levels and glucose intolerance. As a result, we found a negative association between serum creatinine levels, which increased with additional exercise, and the onset of IFG in both sexes after adjusting for variables previously reported to be risk factors for the onset of glucose intolerance and factors associated with chronic kidney disease.

After adjusting for age, sex, race, height, physical activity, and neuropathy, an association was found between increased levels of glucose and insulin after an oral glucose tolerance test (OGTT) and lower muscle mass (estimated using the mid-thigh cross-sectional area) in the Baltimore Longitudinal Study of Aging, which involved 587 participants without diabetes (21). The authors of that study concluded that glucose and insulin levels likely had an early effect on the muscle mass, even in the absence of diabetes (21). Meanwhile, the Health, Aging, and Body Composition Study, which involved 2,166 older adults without diabetes at baseline over a median follow-up period of 11.3 years, investigated the association between muscle quantity and strength and incident diabetes, and whether this association differed according to

Table 2. Results of the Univariate Analyses of Risk Factors for the Onset of Impaired Fasting Glucose*.

\begin{tabular}{|c|c|c|c|c|c|}
\hline & & \multicolumn{2}{|l|}{ Men } & \multicolumn{2}{|l|}{ Women } \\
\hline & & $\mathrm{HR}(95 \% \mathrm{CI})$ & $\mathrm{p}$ value & $\mathrm{HR}(95 \% \mathrm{CI})$ & $\mathrm{p}$ value \\
\hline Age & (years) & $1.07(1.05-1.09)$ & $<0.001$ & $1.096(1.07-1.13)$ & $<0.001$ \\
\hline Body mass index & $\left(\mathrm{kg} / \mathrm{m}^{2}\right)$ & $1.15(1.11-1.19)$ & $<0.001$ & $1.25(1.19-1.31)$ & $<0.001$ \\
\hline Systolic blood pressure & $(\mathrm{mmHg})$ & $1.03(1.02-1.04)$ & $<0.001$ & $1.03(1.02-1.04)$ & $<0.001$ \\
\hline Alanine aminotransferase & $(\mathrm{U} / \mathrm{L})$ & $1.011(1.008-1.014)$ & $<0.001$ & $1.02(1.01-1.03)$ & 0.004 \\
\hline Serum creatinine & $(\mu \mathrm{M})$ & $0.978(0.96-0.99)$ & 0.0011 & 0.967 (0.937-0.999) & 0.04 \\
\hline Uric acid & $(\mu \mathrm{M})$ & $1.004(1.002-1.005)$ & $<0.001$ & $1.012(1.007-1.016)$ & $<0.001$ \\
\hline Fasting plasma glucose & $(\mathrm{mM})$ & $43.9(28.0-69.5)$ & $<0.001$ & $66.9(36.1-127.2)$ & $<0.001$ \\
\hline Total cholesterol & $(\mathrm{mM})$ & $1.33(1.14-1.54)$ & $<0.001$ & $1.71(1.35-2.13)$ & $<0.001$ \\
\hline Triacylglycerol & $(\mathrm{mM})$ & $1.27(1.21-1.33)$ & $<0.001$ & $1.52(1.30-1.68)$ & $<0.001$ \\
\hline Alcohol drinkers & $(\%)$ & $1.34(0.996-1.79)$ & 0.053 & $1.94(1.08-4.12)$ & 0.156 \\
\hline Current smokers & $(\%)$ & $1.41(1.08-1.86)$ & 0.013 & $1.37(0.48-3.07)$ & 0.518 \\
\hline Exercise habit** & $(\%)$ & $0.92(0.68-1.22)$ & 0.565 & $0.76(0.41-1.32)$ & 0.348 \\
\hline Family history of diabetes & $(\%)$ & $1.45(1.04-1.99)$ & 0.029 & $1.84(1.11-2.97)$ & 0.0189 \\
\hline
\end{tabular}

*The onset of impaired fasting glucose was defined as a fasting plasma glucose level $\geq 6.11 \mathrm{mM}$ during the observation period.

**Exercise habit: no exercise or consciousness of exercise vs. periodic exercise.

HR: hazard ratio 
Table 3. Association between Serum Creatinine Levels $(\mu M)$ and Impaired Fasting Glucose Onset*.

\begin{tabular}{cccccc}
\hline & \multicolumn{3}{c}{ Men } & & \multicolumn{2}{c}{ Women } \\
\cline { 2 - 3 } \cline { 5 - 6 } \cline { 5 - 6 } & aHR $(95 \% \mathrm{CI})$ & p value & & aHR $(95 \% \mathrm{CI})$ & p value \\
\hline Model 1 & $0.98(0.96-0.99)$ & 0.008 & & $0.94(0.91-0.97)$ & $<0.001$ \\
Model 2 & $0.98(0.96-0.99)$ & 0.002 & & $0.94(0.91-0.97)$ & $<0.001$ \\
Model 3 & $0.98(0.96-0.99)$ & 0.004 & & $0.967(0.937-0.999)$ & 0.041 \\
\hline
\end{tabular}

Model 1 was adjusted for the variables previously reported to be risk factors for the onset of glucose intolerance and associated with chronic kidney disease, including age (years), body mass index $\left(\mathrm{kg} / \mathrm{m}^{2}\right)$, systolic blood pressure $(\mathrm{mmHg})$, uric acid $(\mu \mathrm{M})$, fasting plasma glucose $(\mathrm{mM})$, exercise habit $(\%)$, and a family history of diabetes $(\%)$.

Model 2 was adjusted for the variables associated with metabolic diseases, including body mass index $\left(\mathrm{kg} / \mathrm{m}^{2}\right)$, systolic blood pressure $(\mathrm{mmHg})$, alanine aminotransferase $(\mathrm{U} / \mathrm{L})$, uric acid $(\mu \mathrm{M})$, fasting plasma glucose $(\mathrm{mM})$, total cholesterol $(\mathrm{mM})$, and triacylglycerol (mM).

Model 3 was adjusted for variables associated with life style, including alcohol consumption (\%), current smoking status (\%), and exercise habit (\%).

*The onset of impaired fasting glucose was defined as a fasting plasma glucose level $\geq 6.11 \mathrm{mM}$ during the observation period.

aHR: adjusted hazard ratio

BMI category (22). The authors found that greater muscle mass (abdominal and thigh muscles) and greater muscle area were predictive of a lower risk of incident type 2 diabetes in normal-weight women (HR, 0.37; 95\% CI, 0.17-0.83) and older normal-weight women (HR, 0.58; 95\% CI, 0.27-1.27), respectively (22). They also found an increased risk of incident type 2 diabetes in overweight and obese women (HR, 1.23; 95\% CI, 0.98-1.54 and HR, 1.28; 95\% CI, 1.00-1.64, respectively). However, no significant associations were found between BMI category and strength, or any other measures in men (all p>0.05). These controversial findings could be related to intermuscular adipose tissue depots in obese women (22). The serum creatinine level reflects the muscle mass independent of intermuscular adipose tissue depots and might therefore be a better marker of the onset of IFG than muscle mass determined via imaging.

The association between serum creatinine levels and the onset of type 2 diabetes among 8,570 Japanese men (age range, 40-55 years) during a 4-year follow-up period was investigated by Harita et al. (18). They demonstrated, through a multiple logistic regression analysis, that lower serum creatinine levels increased the risk of type 2 diabetes [a serum creatinine level of $0.40-0.60 \mathrm{mg} / \mathrm{dL}$ had an odds ratio of 1.91 (95\% CI, 1.44-2.54) relative to a level of 0.71 and 0.80 $\mathrm{mg} / \mathrm{dL}]$ (18). However, in that study, even though the participants might have undergone annual health checkups and received interventions thereafter, the authors only evaluated the participants during two time periods (April 2000-March 2001 and April 2004-March 2005) (18). Moreover, the participants were all men; this relationship was not evaluated in women, even though previous studies have reported sexrelated differences in skeletal muscle mass among diabetes patients $(9,22)$.

Several underlying mechanisms may explain this association between the serum creatinine levels and the onset of
IFG. For example, the loss of muscle mass can reduce the expression and distribution of GLUT4, which could result in a reduction of the insulin-stimulated glucose uptake by skeletal muscle, thereby exacerbating insulin resistance $(23,24)$. Furthermore, in the present study, an association was found between serum creatinine levels and exercise habits. Thus, we thought that the low serum creatinine levels, which might reflect a low muscle volume, were associated with the onset of IFG.

The strengths of the present study include the large sample size and the general population setting. In addition, with the exception of one case in which the BMI value was missing $(0.015 \%)$, the data collection was complete. However, it should be noted that the present study also had a number of limitations. First, only annual data were collected, and these data were not truly continuous. Second, self-reported questionnaires were used, which raises the possibility that the accuracy of our findings could have been affected by misreported data. Third, we did not utilize data from the $75 \mathrm{~g}$ OGTT because the $75 \mathrm{~g}$ OGTT was not conducted during these annual health checkups. Thus, the IFG and normal glucose tolerance groups might have included individuals with impaired glucose tolerance or diabetes. Fourth, the fasting plasma glucose level is affected to a greater extent by gluconeogenesis in the liver and kidney than glucose metabolism disorders of the muscle in the early stage of glucose intolerance, and the effect of glucose intolerance due to muscle mass emerges earlier in the postprandial period than during the fasting period. Thus, to examine the relationship between serum creatinine levels and the onset of glucose intolerance, the examination of postprandial glucose levels was more appropriate. Fifth, we did not evaluate either the muscle mass or the amount of body fat among the subjects. Sixth, subjects who exercised periodically may have a strong attitude to health and may have been more careful with their 
diet. However, we did not assess the meal intake in this study. Seventh, the statistical power of Models 1, 2, and 3 was low (from 0.147 and 0.376), although the creatinine level is a continuous variable. Finally, the study population only included Japanese patients. Thus, to confirm our findings, a prospective validation study should be conducted in various populations because medical practices differ among locations and races.

Despite these limitations, our study reports several noteworthy results. First, a low serum creatinine level appears to be associated with the onset of IFG. Second, serum creatinine levels appear to increase with exercise, which might suggest the importance of exercise therapy. These findings suggest that the measurement of serum creatinine levels could help clinicians identify people at risk of developing type 2 diabetes, and thereby prevent further disease progression.

\section{The authors state that they have no Conflict of Interest (COI).}

\section{Financial Support}

This work was supported in part by a Grant-in-Aid for Scientific Research from the Japan Society for the Promotion of Science (JSPS KAKENHI 15K00874 to T.M., 15K09006 to Y.H.) and a research grant from Ehime University.

\section{References}

1. Sarwar N, Gao P, Seshasai SR, et al.; Emerging Risk Factors Collaboration. Diabetes mellitus, fasting blood glucose concentration, and risk of vascular disease: a collaborative meta-analysis of 102 prospective studies. Lancet 375: 2215-2222, 2010.

2. Hofbauer LC, Brueck CC, Singh SK, Dobnig H. Osteoporosis in patients with diabetes mellitus. J Bone Miner Res 22: 1317-1328, 2007.

3. Pais R, Charlotte F, Fedchuk L, et al.; LIDO Study Group. A systematic review of follow-up biopsies reveals disease progression in patients with non-alcoholic fatty liver. J Hepatol 59: 550-556, 2013.

4. DeFronzo RA, Tripathy D. Skeletal muscle insulin resistance is the primary defect in type 2 diabetes. Diabetes Care 32: S157-S 163, 2009.

5. Katz LD, Glickman MG, Rapoport S, Ferrannini E, DeFronzo RA. Splanchnic and peripheral disposal of oral glucose in man. Diabetes 32: 675-679, 1983.

6. Kraegen EW, James DE, Jenkins AB, Chisholm DJ. Dose-response curves for in vivo insulin sensitivity in individual tissues in rats. Am J Physiol 248: E353-E362, 1985.

7. Volpato S, Bianchi L, Lauretani F, et al. Role of muscle mass and muscle quality in the association between diabetes and gait speed. Diabetes Care 35: 1672-1679, 2012.

8. Park SW, Goodpaster BH, Strotmeyer ES, et al. Decreased muscle strength and quality in older adults with type 2 diabetes: the health, aging, and body composition study. Diabetes 55: 1813-
$1818,2006$.

9. Kim TN, Park MS, Yang SJ, et al. Prevalence and determinant factors of sarcopenia in patients with type 2 diabetes: the Korean Sarcopenic Obesity Study (KSOS). Diabetes Care 33: 1497-1499, 2010.

10. Andrews R, Greenhaff P, Curtis S, Perry A, Cowley AJ. The effect of dietary creatine supplementation on skeletal muscle metabolism in congestive heart failure. Eur Heart J 19: 617-622, 1998.

11. Kim SW, Jung HW, Kim CH, Kim KI, Chin HJ, Lee H. A new equation to estimate muscle mass from creatinine and cystatin $\mathrm{C}$. PLoS One 11: e0148495, 2016.

12. Miyake T, Kumagi T, Furukawa $S$, et al. Hyperuricemia is a risk factor for the onset of impaired fasting glucose in men with a high plasma glucose level: a community-based study. PLoS One 9: e107882, 2014.

13. Chalasani N, Younossi Z, Lavine JE, et al. The diagnosis and management of non-alcoholic fatty liver disease: practice guideline by the American Association for the Study of Liver Diseases, American College of Gastroenterology, and the American Gastroenterological Association. Hepatology 55: 2005-2023, 2012.

14. World Health Organization, International Diabetes Federation. Definition and Diagnosis of Diabetes Mellitus and Intermediate Hyperglycaemia. World Health Organization, Geneva, 2006.

15. Kuzuya T, Nakagawa S, Satoh J, et al. Report of the Committee on the classification and diagnostic criteria of diabetes mellitus. Diabetes Res Clin Pract 55: 65-85, 2002.

16. Matsuo S, Imai E, Horio M, et al. Revised equations for estimated GFR from serum creatinine in Japan. Am J Kidney Dis 53: 982992, 2009.

17. American Diabetes Association. Screening for diabetes. Diabetes Care 25: S21-S24, 2002.

18. Harita N, Hayashi T, Sato KK, et al. Lower serum creatinine is a new risk factor of type 2 diabetes: the Kansai healthcare study. Diabetes Care 32: 424-426, 2009.

19. Sairenchi T, Iso H, Nishimura A, et al. Cigarette smoking and risk of type 2 diabetes mellitus among middle-aged and elderly Japanese men and women. Am J Epidemiol 160: 158-162, 2004.

20. Waki K, Noda M, Sasaki S, et al.; JPHC Study Group. Alcohol consumption and other risk factors for self-reported diabetes among middle-aged Japanese: a population-based prospective study in the JPHC study cohort I. Diabet Med 22: 323-331, 2005.

21. Kalyani RR, Metter EJ, Ramachandran R, Chia CW, Saudek CD, Ferrucci L. Glucose and insulin measurements from the oral glucose tolerance test and relationship to muscle mass. J Gerontol A Biol Sci Med Sci 67: 74-81, 2012.

22. Larsen BA, Wassel CL, Kritchevsky SB, et al. Association of muscle mass, area, and strength with incident diabetes in older adults: the Health ABC Study. J Clin Endocrinol Metab 101: 1847-1855, 2016.

23. Bianchi L, Volpato S. Muscle dysfunction in type 2 diabetes: a major threat to patient's mobility and independence. Acta Diabetol 53: 879-889, 2016.

24. Phielix E, Mensink M. Type 2 diabetes mellitus and skeletal muscle metabolic function. Physiol Behav 94: 252-258, 2008.

The Internal Medicine is an Open Access journal distributed under the Creative Commons Attribution-NonCommercial-NoDerivatives 4.0 International License. To view the details of this license, please visit (https://creativecommons.org/licenses/ by-nc-nd/4.0/).

(C) 2019 The Japanese Society of Internal Medicine Intern Med 58: 505-510, 2019 